\title{
Review: long term effectiveness of non-drug treatments for sleep problems in young children is more evident
}

Ramchandani $P$, Wiggs $L$, Webb Vet al. A systematic review of treatments for settling problems and night waking in young children. BMJ 2000 Jan 22;320:209-13.

\section{QUESTION: In healthy, young children, are drug and non-drug treatments effective for settling problems and night waking?}

\section{Data sources}

Studies were identified by searching Medline (1966 to September 1998), EMBASE/Excerpta Medica (1980 to June 1998), PsycLIT (1974 to September 1998), Biological Abstracts (1985 to June 1998), CINAHL (1982 to September 1998), SIGLE (1980 to June 1998), and the Cochrane database (issue 2, 1998); scanning the bibliographies of identified articles and those in reviews and books; handsearching the Journal of Child Psychology and Psychiatry; and contacting authors and manufacturers of trimeprazine.

\section{Study selection}

Studies were included if they were randomised controlled trials (describing drug or non-drug interventions) of young children ( $\leqslant 5$ y of age) with an established sleep or night waking problem that had an outcome measure of number of night wakings, time to settle, or number of nights in which these problems occurred.

\section{Data extraction}

Data were extracted on the study design, number and age range of the participants, referral source, sleep problem, intervention and control procedures, outcome scores and measures, and assessment times.

\section{Main results}

9 studies met the selection criteria: 4 drug trials, 4 on behavioural treatments, and 1 on a general nondirective educational approach. A meta-analysis was not done because of study heterogeneity. 2 drug trials compared trimeprazine (maximum dose $90 \mathrm{mg}$ ) with placebo. Both showed a statistically significant positive effect of trimeprazine in the short term. Some children treated with trimeprazine, however, continued to wake at night, and up to one third did not improve with trimeprazine. Long term results were less favourable. 1 trial compared niaprazine with chlordesmethyl diazepam and found the drugs to be similarly effective. The fourth drug trial compared trimeprazine as an adjunct to an extinction programme with placebo and found no difference between the groups at end of treatment and at 4 weeks of follow up.

The non-drug interventions investigated were positive routines, graduated extinction, scheduled wakings, extinction or systematic ignoring, modified extinction, an educational booklet, and a sleep programme. In both the short and long term, scheduled wakings and extinction were more effective in reducing night waking than was control treatment (a sleep diary), and positive routines and graduated extinction were more effective in reducing settling problems than was a waiting list. A non-directive educational booklet showed no benefit over a control procedure. The benefits of a modified extinction procedure and of a booklet giving specific advice were unclear because no untreated control group comparisons were done.

\section{Conclusion}

Drug and some non-drug treatments are effective in the short term for reducing settling problems and night waking in young children, but long term beneficial effects are more evident with non-drug treatments.

\section{COMMENTARY}

Treatment for sleep problems in young children varies considerably by geographic area. For example, in a study by Kopferschmitt $e t a l,{ }^{1}$ it was reported that some $12 \%$ of the 11595 children enrolled in kindergarten in the Bas Rhin province in France had been given psychotropic medication for "sleep problems" for more than 6 months and up to 5 years. In contrast, medication for sleep problems is rarely if ever given in the US, but US practitioners show great interest in the effectiveness of various behavioural approaches for sleep difficulties; these approaches have been well documented in a recent review article. ${ }^{2}$

The review by Ramchandani et al teaches us 4 lessons. Firstly, drugs can have a short term effect on sleep behaviour, but little evidence exists to suggest they have a long term effect. One reason for this finding is that sleep problems are often a reflection of a specific parental management style, ${ }^{3}$ which governs a child's day and night behaviour. This type of problem will not be modified by a sleeping pill. Secondly, many parents will not allow their young children to receive psychotropic drugs; hence, studies of drug use for childhood sleep problems may use selective and potentially unrepresentative populations. Thirdly, falling asleep and the number of night wakings are influenced by the neurobehavioural development of the infant, especially during the first 6 months. Thus, $75 \%$ of children 6 months of age do not disturb their parents during the night, whereas virtually all do so earlier. Fourthly, night waking is considered normal in infants. The concern over night waking occurs when children "call out" on waking rather than soothing themselves back to sleep. It is this ability that treatment needs to develop, a modification of behaviour that cannot be accomplished in the long term with medication.

Klaus Minde, $\mathrm{MD}$ The Montreal Children's Hospital Montreal, Quebec, Canada

1 Kopferschmitt J, Meyer P, Jaege A, et al. Sleep disorders and use of psychotropic drugs in 6-year-old children. Rev Epidemiol Sante Publique 1992;40:467-71.

2 Mindell JA. Empirically supported treatments in pediatric psychology: bedtime refusal and night wakings in young children.J Pediatr Psychol 1999;24:465-81.

3 Minde K, Popiel K, Leos N, et al. The evaluation and treatment of sleep disturbances in young children. $J$ Child Psychol Psychiatry 1993;34:521-33. 